\title{
POSITRON TRAPPING MODEL IN FINE GRAINED SAMPLE
}

\begin{abstract}
J. DRYZEK
Institute of Nuclear Physics, Radzikowskiego 152, 31-342 Kraków, Poland

In the paper we consider the positron trapping model in the sample where the spherical particles were immersed in the medium. The radius of the particles was small enough to take into account the diffusion of positrons. We present the exact solutions of the model for two cases, when transition of the positrons from the particle to the medium was neglected and when it was taken into consideration. The theoretical calculations were applied to description of the positron annihilation in the copper sample.where the recrystallization process took place.
\end{abstract}

PACS numbers: $78.70 . \mathrm{Bj}$

\section{Introduction}

We observe the continuous progress in the designing of the nanostructure, powdered and composite materials which offer new properties and/or features. It indicates studies their microstructure. Positron annihilation spectroscopy as a suitable tool for the study of the electronic and atomic structure or open volume defects should be included in such studies too. Nevertheless, for obtaining the quantitative information in these materials, a clear interpretation of positron experiments is needed.

The experimental results of the measurements of the positron lifetime spectroscopy, Doppler broadening of annihilation line and angular correlation of annihilation radiation are interpreted in the literature using the standard trapping model (STM) [1]. In this model the population of positrons implanted in a sample is expressed as a sum of exponential decays. When in the sample two phases exist the population of positrons can be written as follows:

$$
n(t)=I_{1} \exp \left(-\lambda_{1} t\right)+I_{2} \exp \left(-\lambda_{2} t\right)
$$

where the annihilation rate $\lambda_{1,2}$ is the inverse of the positron lifetime, $\lambda_{1,2}=1 / \tau_{1,2}$ and $I_{1,2}$ can be associated with the volume fraction of these phases, respectively. The useful quantity which can be deduced from (1) is the average positron lifetime

$$
\bar{\tau}=\int_{0}^{\infty} n(t) \mathrm{d} t=\frac{I_{1}}{\lambda_{1}}+\frac{I_{2}}{\lambda_{2}} .
$$

This model also in version with defects, which localize positrons, neglects the random walk of positrons before the annihilation. The positron diffusion cannot be 
negligible if the distance between phases or their spatial size are comparable with the positron diffusion length: $L_{+}=\sqrt{D_{+} \tau}$, where $D_{+}$is the positron diffusion coefficient and $\tau$ is the positron lifetime. This quantity is of order of $0.1 \mu \mathrm{m}$. From this one can conclude the strong limitation of the STM which rather should not be applied to the interpretation of positron annihilation results obtained in inhomogeneous, composite or fine grained materials.

The aim of the paper is to present the solution of the so-called diffusion trapping model (DTM) equations for the case which assumes the small spherical particles immersed into the medium. This may help in understanding phenomena when positrons annihilate in composite or inhomogeneous materials.

\section{The theory}

Let us consider the spherical particles of radius $R$ which are randomly uniformly distributed inside the medium (Fig. 1). The total volume of particles is $V_{1}$ and of medium $V_{2}$. At $t=0$ all positrons are also uniformly distributed in a sample and the annihilation rate for positrons in the particle is equal to $\lambda_{1}$ and in the medium $\lambda_{2}$. In the first approach, let us assume that inside the particles positrons

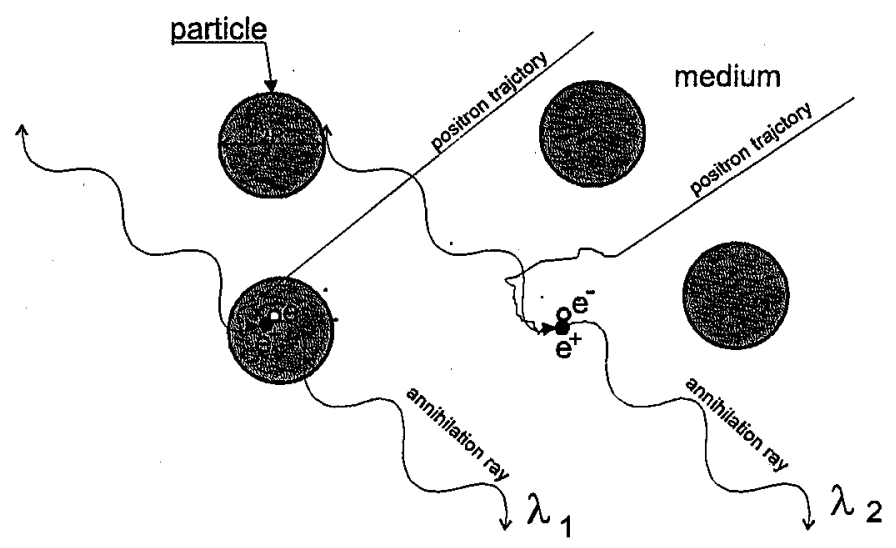

Fig. 1. The model of the spherical particles immersed into the medium.

annihilate faster than in the medium, $\lambda_{1}>\lambda_{2}$. The radius of the particle is small enough to take into account the diffusion of positrons, so their concentration is described by the diffusion equation as follows:

$$
\frac{\partial C(r, t)}{\partial t}=D_{+} \nabla^{2} C(r, t)-\lambda_{1} C(r, t)
$$

where $D_{+}$is the positron diffusion coefficient in the particle. For simplification let us assume that the total number of positrons in the medium is described by the rate equation

$$
\frac{\mathrm{d} n_{2}(t)}{\mathrm{d} t}=-\lambda_{2} n_{2}(t)
$$

where no diffusion is taken into account. In this approach we assumethat the 
concentration of positrons at the surface of a particle is equal to their average concentration in the medium

$$
\frac{n_{2}(t)}{V_{2}}=\left.C(r, t)\right|_{|r|=R}
$$

and positrons do not pass from particles to the medium. For solution of the model we will search the total number of positrons

$$
n(t)=n_{2}(t)+\iiint_{V_{1}} C(r, t) \mathrm{d} V .
$$

From this it is easy to deduce the positron lifetime spectrum $-\frac{1}{n(0)} \frac{\mathrm{d} n(t)}{\mathrm{d} t}$, and the positron mean time defined in (2).

We will solve Eqs. (3) and (4) with (5) by application of the Laplace transform. The details of the method were presented e.g. in [2] but now we present the final result i.e., the Laplace transform of a function which represents the total number of positrons (6):

$$
\begin{aligned}
\tilde{n}(s) & =\frac{1-\delta}{s+\lambda_{2}} \\
& +\delta\left[\frac{1}{s+\lambda_{1}}+\frac{3}{\sqrt{s / \lambda_{1}+1}} \frac{\lambda_{1}-\lambda_{2}}{\left(s+\lambda_{1}\right)\left(s+\lambda_{2}\right)} \frac{L_{+}}{R} L\left(\sqrt{s \lambda_{1}+1} \frac{R}{L_{+}}\right)\right],
\end{aligned}
$$

where $L(z)=\operatorname{cth}(z)-1 / z$ is the Langevin function and $\delta=V_{1} /\left(V_{1}+V_{2}\right)$ is the volume fraction of particles. $L_{+}$represents here and below the positron diffusion length in the particle. Unfortunately, the direct inverse of the Laplace transform of Eq. (7) is not possible so we can perform it only in the approximate way in order to get any analytical form. Let us note that if $R \gg L_{+}, L \approx 1$ and finally

$$
\begin{aligned}
n(t) & \approx(1-\delta) \mathrm{e}^{-\lambda_{2} t} \\
& +\delta\left[\mathrm{e}^{-\lambda_{1} t}+\frac{6}{\pi} \frac{L_{+}}{R} \sqrt{\lambda_{1}}\left(\lambda_{1}-\lambda_{2}\right) \int_{0}^{t} \mathrm{e}^{-\lambda_{2} u} \mathrm{e}^{-\lambda_{1}(t-u)} \sqrt{t-u} \mathrm{~d} u\right] .
\end{aligned}
$$

The obtained result only for $R \rightarrow \infty$ tends to the exponential sum (1), for other $R$ it is much more complicated function. From Eq. (7) we can deduce the average positron lifetime as

$$
\bar{\tau}=\tilde{n}(0)=\frac{1}{\lambda_{2}}+\delta\left(\frac{1}{\lambda_{1}}-\frac{1}{\lambda_{2}}\right)\left[1-3 \frac{L_{+}}{R} L\left(\frac{R}{L_{+}}\right)\right],
$$

which depends upon the particle radius which is in opposition to the results of the STM. The closed-form expression predicts a sigmoidal behavior of the mean positron lifetime as a function of the ratio $R / L_{+}$(Fig. 2). The volume fraction $\delta$ affects $\bar{\tau}$ as well. From Eq. (9) we can deduce the value of the $S$-parameter extracted from the Doppler broadened annihilation line. This parameter is defined as the ratio of the central part to the total area under the annihilation line, so finally

$$
S=S_{2}+\delta\left(S_{1}-S_{2}\right)\left[1-3 \frac{L_{+}}{R} L\left(\frac{R}{L_{+}}\right)\right],
$$

where $S_{1,2}$ represent the values of the $S$-parameter associated with positrons which annihilate in the particle and the medium, respectively. 


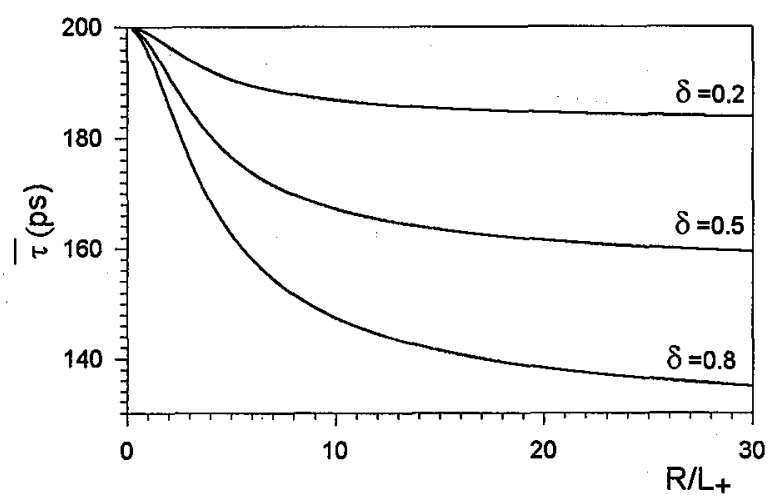

Fig. 2. The mean positron lifetime as a function of the ratio $R / L_{+}$for three values of the volume fraction of the particles, relation (9). In the model it was assumed that the annihilation rate of positrons inside the particles $\left(\lambda_{1}=1 / 110 \mathrm{ps}\right)$ is larger than in the medium $\left(\lambda_{2}=1 / 200 \mathrm{ps}\right)$.

In the presented above calculation we did not consider the explication of the transition of positrons from the particle to the medium. In the second approach we introduce the parameter which will describe these phenomena. Let us assume that the annihilation rate of positrons inside the particle is lower than in the medium: $\lambda_{2}>\lambda_{1}$. The transition rate of positrons from the particles to the medium will be described by the $\alpha$ parameter. In such a case the number of positrons in the medium is given by the equation

$$
\frac{\mathrm{d} n_{2}(t)}{\mathrm{d} t}=-\lambda_{2} n_{2}(t)+\alpha \oiint_{\Sigma} C(r, t) \mathrm{d} S,
$$

where $\Sigma$ is the surface of the particle. Inside the particle the concentration of positrons is expressed by Eq. (3). On the surface of the particle the following constraint must be fulfilled:

$$
D_{+} \oiint_{\Sigma} \nabla C(r, t) \cdot \mathrm{d} S+\alpha \oiint_{\Sigma} C(r, t) \mathrm{d} S=0
$$

After application of the Laplace transform and some algebra we obtain the following relation:

$$
\begin{gathered}
\tilde{n}(s)=\frac{1-\delta}{\lambda_{2}+s}+\delta\left[\frac{1}{\lambda_{1}+s}\right. \\
\left.+\frac{3}{\sqrt{s / \lambda_{1}+1}} \frac{\lambda_{1}-\lambda_{2}}{\left(\lambda_{1}+s\right)\left(\lambda_{2}+s\right)} \frac{L_{+}}{R} \frac{L\left(\sqrt{s / \lambda_{1}+1} \frac{R}{L_{+}}\right)}{1+\frac{L_{+} \lambda_{1} \sqrt{s / \lambda_{1}+1}}{\alpha} L\left(\sqrt{s / \lambda_{1}+1} \frac{R}{L_{+}}\right)}\right] .
\end{gathered}
$$

As before, we are able to find the inverse Laplace transform of this equation only for $R / L_{+} \gg 1$ and $L_{+} \lambda_{1} / \alpha \gg 1$ :

$n(t) \approx\left[1-\delta\left(1-\frac{3 \alpha}{R\left(\lambda_{1}-\lambda_{2}\right)}\right)\right] \mathrm{e}^{-\lambda_{2} t}+\delta\left[1-\frac{3 \alpha}{R}\left(t-\frac{1}{\lambda_{1}-\lambda_{2}}\right)\right] \mathrm{e}^{-\lambda_{1} t}$.

Also this form is not a sum of exponents. 
From (13) we can calculate the average positron lifetime in such a case

$$
\bar{\tau}=\frac{1}{\lambda_{2}}+\delta\left(\frac{1}{\lambda_{1}}-\frac{1}{\lambda_{2}}\right)\left[1-\frac{3 L_{+}}{R} \frac{L\left(R / L_{+}\right)}{1+\frac{L_{+} \lambda_{1}}{\alpha} L\left(R / L_{+}\right)}\right] .
$$

Note that if $\alpha \rightarrow \infty$ this equation tends to Eq. (9) which should be obvious. In this case the increase in the $R / L_{+}$value causes the increase in $\bar{\tau}$ and then saturation to the $1 / \lambda_{2}$ which is presented in Fig. 3.

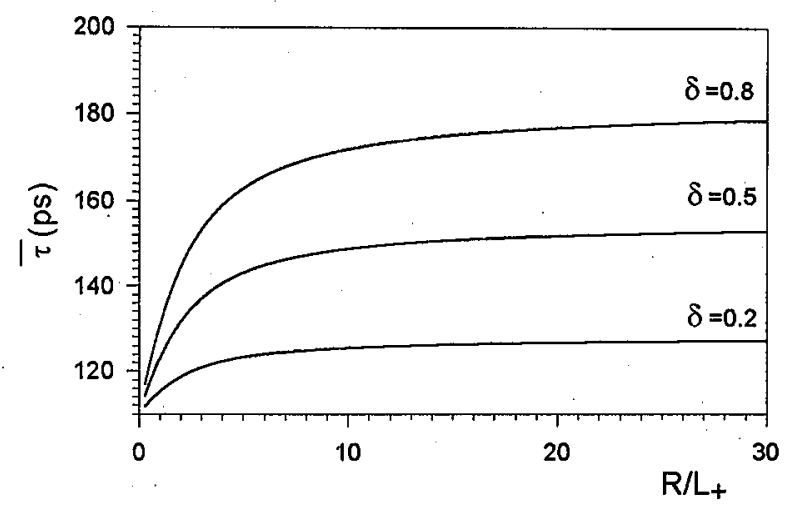

Fig. 3. The mean positron lifetime as a function of the ratio $R / L_{+}$for three values of the volume fraction of the particles, relation (15). In the model it was assumed that the annihilation rate of positrons inside the particles $\left(\lambda_{1}=1 / 200 \mathrm{ps}\right)$ is smaller than in the medium $\left(\lambda_{2}=1 / 110 \mathrm{ps}\right)$. The ratio $L_{+} \lambda_{1} / \alpha$ was equal to 10 .

The value of the $S$-parameter in this approach can be deduced from (15) as follows:

$$
S=S_{2}+\delta\left(S_{1}-S_{2}\right)\left[1-\frac{3 L_{+}}{R} \frac{L\left(R / L_{+}\right)}{1+\frac{L_{+} \lambda_{1}}{\alpha} L\left(R / L_{+}\right)}\right] .
$$

We should emphasize that the presented above dependences of the $\bar{\tau}$ or the $S$-parameter are observed in positron experiments where inhomogeneous samples are studied. As an example we can remind the results of the measurements of the average positron lifetime in alloys, where the precipitation of fine particles occurs (see e.g. [3]). In the next section we will try to apply the presented above relation to description of the recrystallization process observed by positron annihilation method.

\section{The study of the recrystallization process}

In a deformed sample prior to the recrystallization there appears the recovery process which changes the dislocation structure. Because the movement of dislocations causes the creation of certain amount of vacancies or interstitial atoms we postulate that this process involves only small changes in the positron annihilation characteristics. This changes are in opposition to the changes during the recrystallization process when the formation of new strain-free and defects-free grains and the subsequent growth of these with consuming of the deformed microstructure take place. The mechanisms controlling recrystallization are thermally 
activated [4]. The radius of the new grain is the following function of time $t$ and absolute temperature $T$ of the sample:

$$
R^{n}-R_{0}^{n} \propto t \exp \left(-\frac{Q}{k_{\mathrm{B}} T}\right),
$$

$k_{\mathrm{B}}$ is the Boltzmann constant and $R_{0}$ is the initial radius. Because the grain volume increases due to the migration of the boundaries, thus $Q$ represents the activation energy for that process. The value of $n$ parameter depends on the symmetry of the grain, for a sphere it is equal to 2 . The volume of fraction recrystallized increases from 0 to 1 as the transformation proceeds with time $t$ and it is described by the following form:

$$
X_{V}=1-\exp \left(-B t^{m}\right)
$$

which is called the Avrami, Johnson-Mehl equation. The parameter $m$ lies between 3 and 4 . Note that in the sample where recrystallization process takes place, two regions are present in which positron annihilation characteristics are different. In the deformed region where many defects are present the annihilation rate is smaller than in the new grains. Thus Eqs. (17) and (18) can be put into Eqs. (9) or (10) for description of the change of the average positron mean lifetime or the $S$-parameter during the recrystallization process. Both parameters are functions of time and temperature.

In our experiment we measured the peak height of the angular correlation of annihilation radiation curve which was sensitive to the presence of open-volume defects and directly corresponds to the value of the $S$-parameter. The spectrometer had two arms fixed at $180^{\circ}$ with scintillator detectors behind the long slits on each arm. The slits were equal to $3.3 \mathrm{mrad}$. The detectors observed the sample located between the slits, lit with positrons emitted from the ${ }^{22} \mathrm{Na}$ source. In the experiment the number of coincidence events was measured vs. temperature of the sample [5]. We used the single crystal of copper (99.9\% purity) which was rolled in the direction [111] and the thick was reduced by about $83 \%$. Figure 4 presents the results of the measurements of the peak height as a function of temperature, each point was measured over two hours at the same temperature. We observed the sudden drop of the measured quantity at $90^{\circ} \mathrm{C}$ which was correlated with the increase in the fraction of recrystallized volume by direct microscopic observation. The shift in the temperature scale could arise because both measurements were performed in the independent way. The dashed line in Fig. 4 presents the results of the best fit of Eq. (9) where the radius $R$ was expressed by the relation (17). From this the value of the activation energy $Q$ was estimated as equal to $1.18 \pm 0.02 \mathrm{eV}$. In this approach $\delta=1$ which can be an approximation of the real process. In the next approach we can assume that the volume of fraction of recrystallized grains is a function of temperature as follows: $\delta=1+0.022\left(T\left[{ }^{\circ} \mathrm{C}\right]-118.85\right)$, for $73.4^{\circ} \mathrm{C}<$ $\mathrm{T}<118.85^{\circ} \mathrm{C}$. This approximate relation was deduced from the experiment. After application of this relation together with relation (17) in Eq. (9) one can obtain much better agreement with the experiment. The solid line in Fig. 4 presents the best fit of such an approach, the activation energy $Q$ was estimated as equal to $1.1 \pm$ $0.3 \mathrm{eV}$. Note that the obtained value of the activation energy is close to the value of the activation energy for migration of high angle grain boundary in copper reported 


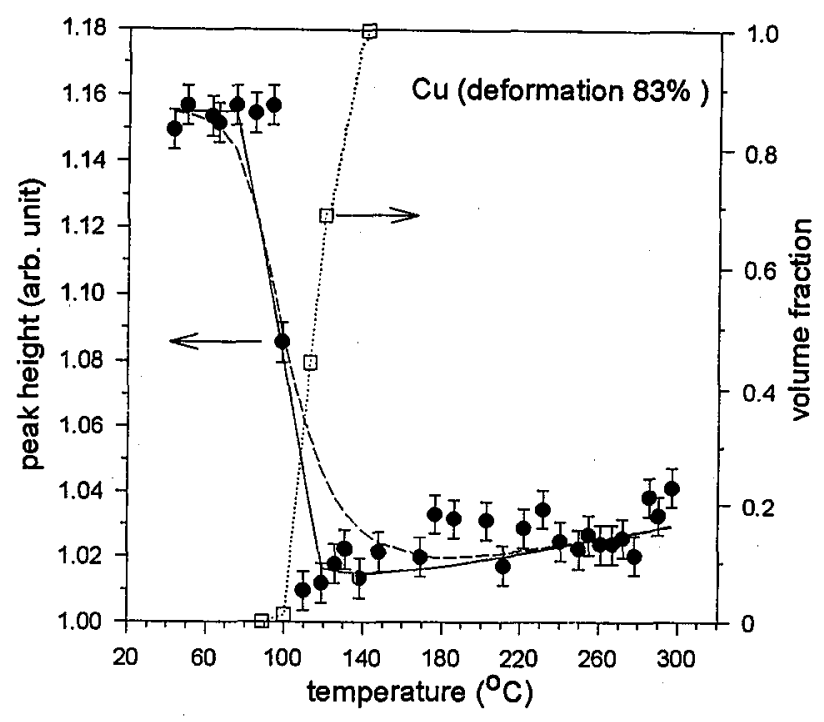

Fig. 4. The peak height of angular correlation of annihilation radiation versus the temperature measured in the copper single crystal after deep plastic deformation, the closed points. The open points represent the volume fraction of the recrystallized area. The solid and dashed lines represent the best fit of the assumed model, see text.

in the literature: $1.25 \mathrm{eV}$ and measured also in the recrystallization experiments during the direct observation [4]. The good coincidence of this value with the value of the activation energy $Q$ obtained in the positron experiments shows that the diffusion trapping model can be helpful also in obtaining the quantitative information about the processes in the inhomogeneous samples.

\section{Acknowledgments}

The author would like to thank the Committee for Scientific Research (Poland) for supporting this work under research grant No. 2P03B 02710 and Dr. M. Wróbel for preparation of the samples.

\section{References}

[1] W. Brandt, in: Positron Annihilation, Eds. A.T. Steward, O. Roellig, Academic Press, New York 1967, p. 155. See also W. Brandt, Appl. Phys. 5, 1 (1974).

[2] R. Würschum, A. Seeger, Philos. Mag. A 73, 1489 (1996).

[3] R. Krause, G. Dlubek, A.t. Beresina, in: Positron Studies of Defects, Eds. G. Dlubek, O. Brümmer, G. Brauer, K. Hennig, Wissenschaftlische Beiträge, Martin-Luter-Universität, Halle 1987, PL7.

[4] F.J. Humphreys, M. Hatherly, Recrystallization and Related Annealing Phenomena, Pergamon 1996, p. 96.

[5] J. Dryzek, Acta Univ. Wratislav. 1204, 73 (1989). 